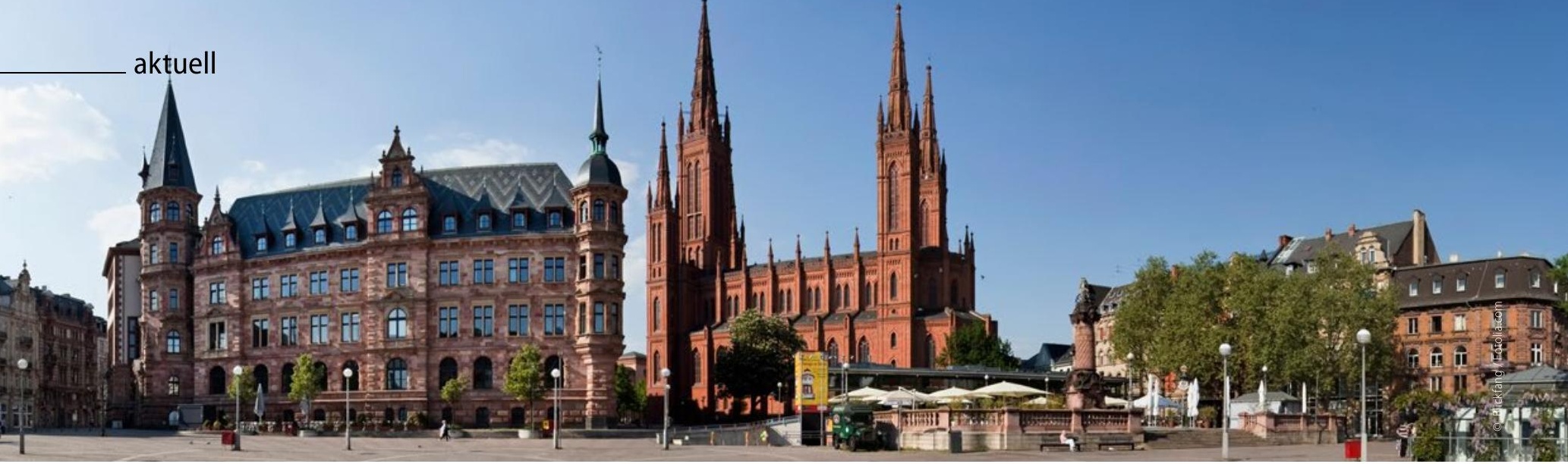

\title{
Rege Beteiligung spricht für das Konzept
}

Vor sechs Jahren wurde das Psychiatrie Update zum ersten Mal angeboten. Da die Fortbildung den aktuellen Stand der Forschung in ihrem Fachgebiet gut abbildet, gehört sie inzwischen zu den fixen Terminen im Kongresskalender vieler Psychiater aus Klinik und Praxis. Diesmal hatten die Referenten die wichtigsten Publikationen des zurückliegenden Jahres aus 14 Themenschwerpunkten der Psychiatrie praxisrelevant aufbereitet.

\section{„Personal space“ bei Psychosen}

\begin{abstract}
Die nicht verbale Kommunikation ist in der Psychiatrie eine Quelle für fundamentale Missverständnisse. Die in einer Studie zur Wohlfühldistanz bei Psychosen gewonnenen Erkenntnisse sind deshalb direkt relevant für den therapeutischen Umgang mit den Patienten.
\end{abstract}

Trotz ihrer anerkannten Bedeutung in der Psychiatrie ist die nicht verbale Kommunikation noch wenig erforscht, erklärte Professor Werner Strik, Direktor der Universitätsklinik für Psychiatrie und Psychotherapie, Bern. Sie umfasst neben indirekten Signalen und Symbolen (z. B. Körperpflege, Gesundheitsund Ernährungszustand, Kleidung, Schmuck) den emotionalen Ausdruck (z. B. Mimik, Prosodie, vegetative Signale etc.) und die Körperhaltung sowie intentionale Bewegungen. Auch wenn erfahrenes Fachpersonal das unterschiedliche Bedürfnis seiner Patienten nach Nähe und Distanz intuitiv respektiert, komme es immer wieder zu fundamentalen - und teils gefährlichen Missverständnissen, so Strik. Beispielsweise wenn sich psychotische Patienten durch räumliche Nähe bedrängt fühlen und ausweichen oder aber aggressiv werden. Seine Arbeitsgruppe führte deshalb eine Studie zur Toleranz räumlicher Nähe bei schizophrenen Patienten durch und nutzte dazu die Berner Psychopathologie-Skala für Psychosen (BPS) sowie objektive Distanzmessungen [Schoretsanitis G et al. Eur Psychiatry 2015; 31: 1 -7]. Wie Strik erläuterte, kann mit der BPS die paranoide Symptomatik in "paranoid power", das heißt „positive“ emotionale Dysregulation in Form von abnormen Machtgefühlen von „paranoid threat" in Form von Bedrohungsgefühlen unterschieden werden. Die Untersuchung deckte auf, dass sich Patienten mit paranoiden Bedrohungsgefühlen bereits bei Distanzen unter 2,41 Metern unwohl fühlten. Bei Patienten mit abnormen Machtgefühlen war die Wohlfühldistanz dagegen sogar niedriger als bei gesunden Kontrollpersonen (1,1 Meter).

Die Grenze von 1,1 Meter der gesunden Kontrollpersonen und affektiv nicht auffälligen schizophrenen Patienten war laut Strik auf fast diagnostischem Niveau spezifisch $(83 \%)$ und sensitiv (93\%). „Die Ergebnisse dieser schönen Studie haben direkte Relevanz für den Umgang mit unseren Patienten. Sie liefern nicht verbale Hinweise auf latente paranoide Bedrohungsgefühle, helfen uns aber auch, das Risiko für körperliche Angriffe bei verbalen Respektlosigkeiten besser einschätzen zu können“, erklärte Strik.

\section{Mehr Platz - weniger Zwangsmedikationen}

Angesichts der Erkenntnisse zur persönlichen Distanz müssen laut Strik auch die aktuellen räumlichen Gegebenheiten in psychiatrischen Kliniken hinterfragt werden. Sie sind veraltet und bei weitem zu eng. Dazu präsentierte er interessante Beobachtungen nach dem Umzug der Psychiatrischen Universitätsklinik in Tübingen in einen Neubau im Jahr 2011 [Dresler T et al. World Psychiatry 2015; 14 (1): 105 - 6]: Größere Räume auf mehr Grundfläche mit größeren Fenstern, höchstens Zwei-Bett-Zimmer anstelle von Vier-Bett-Zimmern und großzügigere Rückzugsmöglichkeiten für die Patienten führten dazu, dass in den folgenden Jahren die Zahl und Dauer der Bewegungseinschränkungen und Zwangsmedikationen um $50 \%$ bis $85 \%$ reduziert werden konnten.

Ute Ayazpoor, freie Medizinjournalistin 